\title{
On Massera's theorem concerning the uniqueness of a periodic solution for the Liénard equation. When does such a periodic solution actually exist?
}

Lilia Rosati and Gabriele Villari*

\author{
"Correspondence: \\ villari@math.unifi.it \\ Dipartimento di Matematica \\ 'U. Dini', Università degli Studi di \\ Firenze, viale Morgagni 67/A, \\ Firenze, 50134, Italy
}

\begin{abstract}
In this note we consider the classical Massera theorem, which proves the uniqueness of a periodic solution for the Liénard equation

$$
\ddot{x}+f(x) \dot{x}+x=0,
$$

and investigate the problem of the existence of such a periodic solution when $f$ is monotone increasing for $x>0$ and monotone decreasing for $x<0$ but with a single zero, because in this case the existence is not granted. Sufficient conditions for the existence of a periodic solution and also a necessary condition, which proves that with this assumptions actually it is possible to have no periodic solutions, are presented.

MSC: $34 \mathrm{C} 05 ; 34 C 25$
\end{abstract}

Keywords: Liénard equation; limit cycles; Massera theorem

\section{Preliminaries and well-known results}

The problem of the existence and uniqueness of a periodic solution for the Liénard equation

$$
\ddot{x}+f(x) \dot{x}+x=0
$$

has been widely investigated in the literature. Among the uniqueness results, a special place is, without any doubt, taken by the classical Massera theorem. This is due to the simple geometrical ideas and the fact that this result, despite several efforts, is in most cases no more valid for the generalized Liénard equation

$$
\ddot{x}+f(x) \dot{x}+g(x)=0 .
$$

For related results concerning the more general equation

$$
\ddot{x}+f(x, \dot{x}) \dot{x}+x=0 \text {, }
$$

we refer to [1].

( 2013 Rosati and Villari; licensee Springer. This is an Open Access article distributed under the terms of the Creative Commons Attribution License (http://creativecommons.org/licenses/by/2.0), which permits unrestricted use, distribution, and reproduction in any medium, provided the original work is properly cited. 
Equation (1.1) is equivalent to the phase-plane system

$$
\left\{\begin{array}{l}
\dot{x}=y \\
\dot{y}=-f(x) y-x
\end{array}\right.
$$

and to the Liénard system

$$
\left\{\begin{array}{l}
\dot{x}=y-F(x), \\
\dot{y}=-x,
\end{array} \text { where } F(x)=\int_{0}^{x} f(t) d t .\right.
$$

We present the classical Massera theorem which is a milestone among the results of limit cycles uniqueness for system (1.2).

Theorem 1.1 (Massera [2]) System (1.2) has at most one limit cycle which is stable, and hence equation (1.1) has at most one nontrivial periodic solution which is stable, provided that $f$ is continuous and

1. $f(x)$ is monotone decreasing for $x<0$,

2. $f(x)$ is monotone increasing for $x>0$.

The theorem of Massera improved a previous result due to Sansone [3] in which there was the additional assumption $|f(x)|<2$. This assumption comes from the fact that Sansone was using the polar coordinates. Such a strong restriction on $f$ is clearly not satisfied in the polynomial case, and hence Massera's result is much more powerful. We recall the recent papers [4] and [5] in which a discussion concerning these two results, as well as related results, may be found.

We observe that in his paper Massera was proving the uniqueness of limit cycles, regardless of the existence, because only the monotonicity properties and the continuity were required. It is well known that in order to fulfill the necessary conditions for the existence of limit cycles, the only two cases to be considered are the following:

A $f(x)$ has two zeros $a<0<b$. In this case the existence of limit cycles is granted (see, for instance, [6]). Moreover, $F(x)$ has three zeros at $\alpha<0,0$ and $\beta>0, x F(x)$ is negative for $\alpha<x<\beta(x \neq 0)$ and positive outside this interval, and $F$ is monotone increasing for $x<\alpha$ and $x>\beta$.

B $f(x)$ remains negative for $x<0$ (or for $x>0$ ), while it intersects the $x$-axis once in $x>0$ (or for $x<0$ ).

This is because if we consider the distance from the origin, namely $H(x, y)=x^{2}+y^{2}$, and evaluate $\dot{H}(t)$ in systems (1.2) and (1.3), we get

$$
\begin{aligned}
& \dot{H}(t)=-2 f(x) y^{2} \quad \text { in (1.2), } \\
& \dot{H}(t)=-2 x F(x) \quad \text { in (1.3). }
\end{aligned}
$$

Hence the distance from the origin increases if $f$ is negative and decreases if $f$ is positive, and a necessary condition for the existence of limit cycles is that $f(x)$ changes sign. 
As for [A], in a very recent paper [5], the monotonicity assumptions, which are required in the whole line, were relaxed to the interval $(\alpha, \delta)$, where $\alpha$ and $\delta$ can be easily determined. In particular, the following result holds.

Theorem 1.2 ([5]) The Liénard system (1.3) has exactly one limit cycle, which is stable, provided that $f$ is continuous and

1. if $|\alpha|>\beta, f(x)$ is monotone decreasing for $\alpha<x<0, f(x)$ is monotone increasing for $0<x<\delta$;

2. if $|\alpha|<\beta, f(x)$ is monotone decreasing for $\delta_{1}<x<0, f(x)$ is monotone increasing for $0<x<\beta$ with

$$
\delta=\sqrt{\left(1+F(a)+\frac{\alpha^{2}}{2}\right)^{2}+\beta^{2}}, \quad \delta_{1}=-\sqrt{\left(-F(b)+1+\frac{\beta^{2}}{2}\right)^{2}+\alpha^{2}},
$$

where $a, b$ are the zeros of $f(x)$ and $\alpha, \beta$ are the nontrivial zeros of $F(x)$. (If $|\alpha|=\beta$, the uniqueness result comes from classical results due to Levinson-Smith [7] and Sansone [3] and no monotonicity assumptions are required.)

Let us consider case $[\mathrm{B}]$. Now the existence of the limit cycle is no more granted, and, as far as we know, this case was not explicitly investigated in the literature.

The aim of this note is to discuss such a situation. In the next section, we present some sufficient conditions for the existence, and hence the uniqueness, of the limit cycle as well as a necessary condition. Such a condition shows that actually there are situations in which there is no limit cycle despite the monotonicity properties and the fact that $f(x)$ changes sign.

\section{Existence of the limit cycle}

We consider the case

B $f(x)$ remains negative for $x<0$, while it intersects the $x$-axis once in $x>0$.

The case ' $f(x)$ is negative for $x>0$, while it intersects the $x$-axis once in $x<0$ ' is treated in exactly the same way.

At first we work in the phase-plane

$$
\left\{\begin{array}{l}
\dot{x}=y \\
\dot{y}=-f(x) y-x
\end{array}\right.
$$

and we give a constructive result based on the methods used in [8].

Theorem 2.1 The phase-plane system (1.2) has exactly one limit cycle, which is stable, provided that $f$ is continuous and

1. $f(x)$ is monotone decreasing for $x<0$,

2. $f(x)$ is monotone increasing for $x>0$ and has a single zero at $a>0$,

3 .

$$
\lim _{x \rightarrow+\infty} \frac{x}{f(x)}=\alpha<+\infty
$$


4.

$$
\lim _{x \rightarrow-\infty} f(x)=-L>-1
$$

Proof We just notice that the continuity of $f$ guarantees the property of uniqueness for the solutions to the Cauchy problem associated to system (1.2), and therefore the trajectories of such a system cannot intersect. Consider the 0 -isocline of system (1.2), that is, the points in the phase-plane were $\dot{y}=0$ and $\dot{x} \neq 0$.

For these points, we have $y=-\frac{x}{f(x)}$. Let $\Delta$ be the graph of the function $u(x)=-\frac{x}{f(x)}$. Clearly, we get

$$
\lim _{x \rightarrow a+} u(x)=-\infty
$$

and that $u(x)$ is bounded as $x \rightarrow+\infty$. Consider the slope of the trajectories of system (1.2) given by

$$
y^{\prime}=-f(x)-\frac{x}{y} .
$$

In $x>a, u(x)$ is negative and the slope at the points below $\Delta$ is negative. Hence we can select a point $P\left(x_{0}, u\left(x_{0}\right)\right)$, with $x_{0}>a$ and $x_{0}-a$ arbitrarily small, such that $u\left(x_{0}\right)$ is smaller than all the other values of $u(x)$ for $x>x_{0}$. Let $\gamma^{-}(P)$ be a negative semi-trajectory which starts at $P$. Inspection of the slopes shows that such a semi-trajectory does not intersect $\Delta$, and therefore is bounded away from the $x$-axis for $x>x_{0}$. We now consider the positive semi-trajectory $\gamma^{+}(P)$ which starts at $P$. At first we observe that being monotone, $f(x)$ has a limit, $-L \leq 0$, for $x \rightarrow-\infty$. Hence, for $\epsilon>0$, there exists $x_{1}<0$ such that $-L>f(x)>-L-\epsilon$ for every $x<x_{1}$. It is trivial to see that in the strip $x_{1}<x<x_{0}$ the slope is bounded for $|y|>1$, and therefore $\gamma^{+}(P)$ intersects the line $x=x_{1}$ at a point $P_{1}\left(x_{1}, y_{1}\right)$ with $y_{1}<0$, or intersects the $x$-axis in $x<0$. Clearly, we must consider the first situation being our goal to reach the $x$-axis. For $x<x_{1}$, we get

$$
\begin{aligned}
& L<-f(x)<L+\epsilon, \\
& \frac{1}{L+\epsilon}<\frac{1}{-f(x)}<\frac{1}{L}, \\
& \frac{x}{L+\epsilon}>\frac{x}{-f(x)}>\frac{x}{L}, \quad x<x_{1} .
\end{aligned}
$$

This means that in $x<x_{1}, \Delta$ lies between the lines $\frac{x}{L+\epsilon}$ and $\frac{x}{L}$ and hence the 0 -isocline is bounded away from the line $\frac{x}{L+\epsilon}$. Such a line has the slope $\frac{1}{L+\epsilon}$. On the other hand, in $x<x_{1}$ the slope of $\gamma^{+}(P)$ is

$$
-f(x)-\frac{x}{y}<L+\epsilon-\frac{x}{y}<L+\epsilon .
$$

Therefore, if $L+\epsilon<\frac{1}{L+\epsilon}$, eventually $\gamma^{+}(P)$ will intersect the line $\frac{x-x_{1}}{L+\epsilon}-\frac{x_{1}}{f\left(x_{1}\right)}$ at some $\bar{x}<x_{1}$, and hence also $\Delta$ at some $\bar{x}<x<x_{1}$. A straightforward calculation yields that such an inequality holds if $L+\epsilon<1$, and being $\epsilon$ arbitrary, this gives $L<1$, that is, the value of the limit for $x \rightarrow-\infty$ in the assumptions. Now we recall that in $x<0$ the slope at the 


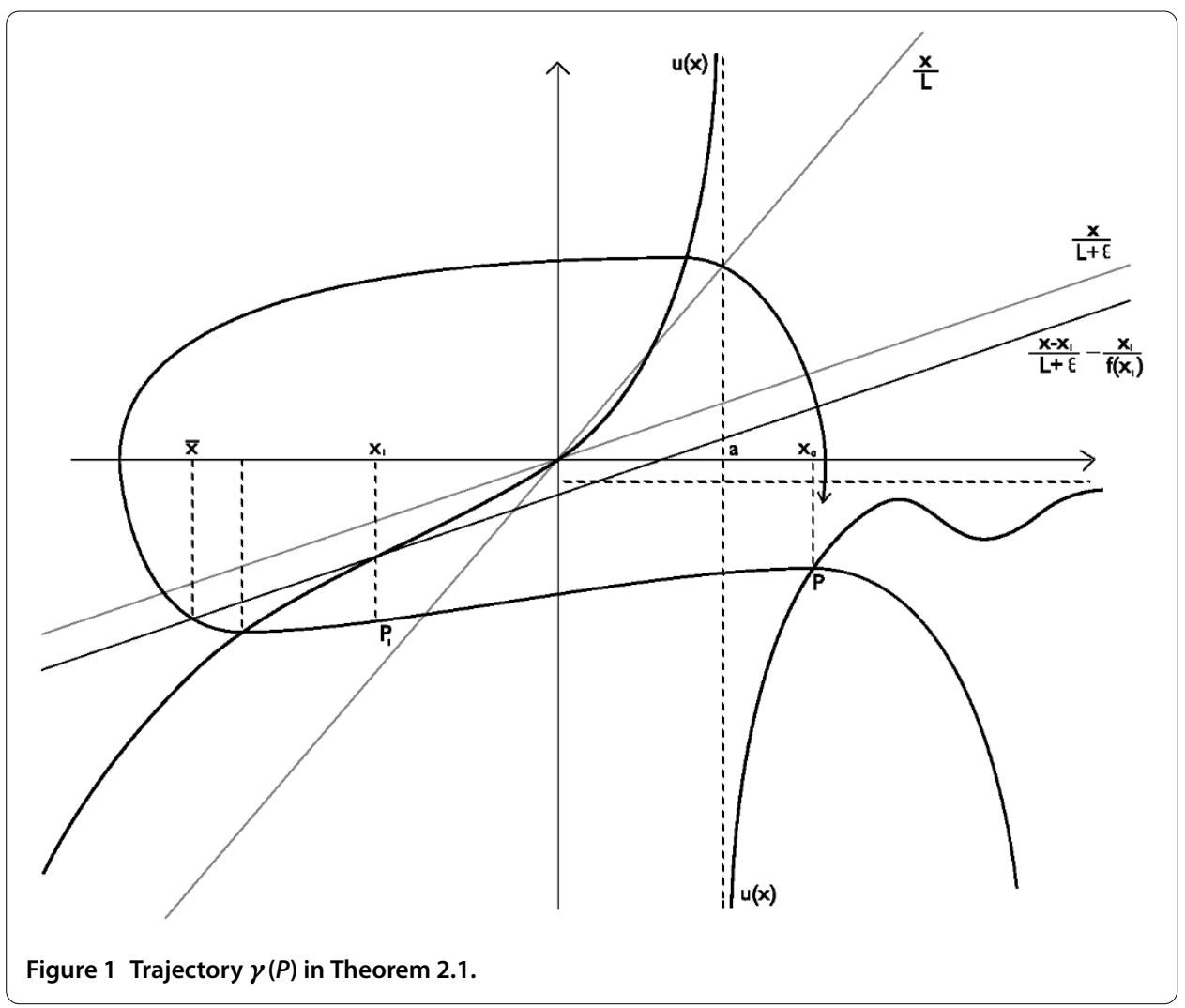

points above $\Delta$ is negative, and it is easy to see that $\gamma^{+}(P)$ eventually will intersect the $x$-axis in $x<0$. In fact, any trajectory intersecting the $x$-axis in $x<0$ intersects the $y$ axis in $y>0$. This is because the slope being bounded for $y>1$, no vertical asymptote is possible. Then $\gamma^{+}(P)$ will intersect the $x$-axis in $x>0$. This proves that the trajectory $\gamma(P)$ is winding (see Figure 1). Being $f(0)<0$, the origin is a source and the existence of a limit cycle is granted by the Poincaré-Bendixson theorem, while the uniqueness is given by monotonicity properties in virtue of the Massera theorem.

We observe that if we keep all the sign assumptions and the limits at infinity, but not the monotonicity, clearly the uniqueness is no more granted, but the existence result still holds, and one can relax the assumption

$$
\lim _{x \rightarrow+\infty} \frac{x}{f(x)}=\alpha<+\infty
$$

with

$$
\lim _{x \rightarrow+\infty} \sup \frac{x}{f(x)}=\alpha<+\infty
$$

as in [8].

Hence we have the following corollary.

Corollary 2.2 The phase-plane system (1.2) has at least a stable limit cycle provided that $f$ is continuous and $f(x)(x-a)>0$, with $a>0$, and 
1.

$$
\lim _{x \rightarrow-\infty} f(x)=-L>-1
$$

2.

$$
\lim _{x \rightarrow+\infty} \sup \frac{x}{f(x)}=\alpha<+\infty
$$

As already mentioned, the first result is based on the direct construction of a winding trajectory.

A more powerful approach can be adopted in the Liénard plane, and it is based on the results presented in [9], where the problem of intersection with the vertical isocline $y=$ $F(x)$ is investigated in full for the more general Liénard system

$$
\left\{\begin{array}{l}
\dot{x}=y-F(x), \\
\dot{y}=-g(x),
\end{array}\right.
$$

where

$$
F(x)=\int_{0}^{x} f(t) d t
$$

where as usual $x g(x)>0$.

The following result holds.

Theorem 2.3 The Liénard system (1.3) has exactly one limit cycle, which is stable, provided that $f$ is continuous and

1. $f(x)$ is monotone decreasing for $x<0$,

2. $f(x)$ is monotone increasing for $x>0$ and has a single zero at $a>0$,

3.

$$
\lim _{x \rightarrow-\infty} f(x)=-L>-2
$$

4.

$$
\lim _{x \rightarrow+\infty} f(x)=M>2
$$

Proof As already mentioned, the continuity of $f$ guarantees the property of uniqueness for the solutions to the Cauchy problem associated to system (1.3), and therefore the trajectories of such a system cannot intersect.

As the proof is based on different applications of both necessary and sufficient conditions for the intersection of a semi-trajectory with the vertical isocline $y=F(x)(2.14)$ given in [9], we are going to introduce the same notation as in [9] and to recall the theorems we are going to use here.

As well as in the phase-plane, for any point $P=\left(x_{0}, y_{0}\right) \in \mathbb{R}^{2}$, we denote by $\gamma^{+}(P)$ and $\gamma^{-}(P)$ the positive, respectively negative, semi-trajectory of the Liénard system (2.13) passing through $P$. With respect to the intersection of $\gamma^{+}(P)$ with the vertical isocline (2.14), 
we observe that by a standard inspection of the direction of the field, it suffices to consider the cases

$$
x_{0} \geq 0, \quad y_{0}>F\left(x_{0}\right)
$$

and

$$
x_{0} \leq 0, \quad y_{0}<F\left(x_{0}\right) .
$$

With respect to the intersection of $\gamma^{-}(P)$ with the vertical isocline (2.14), it suffices to consider the cases

$$
x_{0} \geq 0, \quad y_{0}<F\left(x_{0}\right)
$$

and

$$
x_{0} \leq 0, \quad y_{0}>F\left(x_{0}\right)
$$

As in [9], we define

$$
F_{+}(x):=\max \{0, F(x)\}
$$

and, finally, we introduce the function

$$
\Gamma_{+}(x):=\int_{0}^{x}\left(1+F_{+}(\xi)\right)^{-1} g(\xi) d \xi .
$$

For this particular case, we have the following result.

Theorem 2.4 $\left(\left[9\right.\right.$, Theorem 2.5.]) If, for every $P=\left(x_{0}, y_{0}\right)$ verifying $(2.19), \gamma^{-}(P)$ intersects the curve $y=F(x)$ at $(x, F(x))$ with $x>x_{0}$, then

$$
\limsup _{x \rightarrow+\infty}\left[4 \Gamma_{+}(x)-F(x)\right]=+\infty
$$

Theorem 2.5 ([9, Theorem 2.3.]) If there is a constant $0<\lambda<4$ such that

$$
\lim _{x \rightarrow-\infty}\left[\lambda \Gamma_{+}(x)-F(x)\right]=+\infty
$$

then, for every $P=\left(x_{0}, y_{0}\right)$ verifying $(2.18), \gamma^{+}(P)$ intersects the curve $y=F(x)$ at $(x, F(x))$ with $x<x_{0}$.

Let us see that hypothesis (2.15) implies that there exists $0<\lambda<4$ such that (2.22) is verified. First of all, we observe that in the case we are dealing with $\Gamma_{+}(x)=\int_{0}^{x}\left(1+F_{+}(\xi)\right)^{-1} \xi d \xi$. By hypothesis (2.15), $\forall \epsilon>0$ there exists $x_{1}<0$ such that, for $x<x_{1}$, we have $|f(x)+L|<\epsilon$. It is not restrictive to suppose $L-\epsilon>0$. This is because we are studying the case $L \geq 1$, being 
the result already proved for $L<1$, in virtue of the previous theorem. Then, for $x<x_{1}<0$, we have

$$
\begin{aligned}
& -L+\epsilon>f(x)>-L-\epsilon, \\
& F(x)=F\left(x_{1}\right)+\int_{x}^{x_{1}}-f(x) d x, \\
& 0<F\left(x_{1}\right)+(L-\epsilon)\left(x_{1}-x\right)<F(x)<F\left(x_{1}\right)+(L+\epsilon)\left(x_{1}-x\right) .
\end{aligned}
$$

In our assumptions, for $x<0, F_{+}(x)=F(x)$. Then

$$
\frac{-x}{1+F\left(x_{1}\right)+(L+\epsilon)\left(x_{1}-x\right)}<\frac{-x}{1+F_{+}(x)}<\frac{-x}{1+F\left(x_{1}\right)+(L-\epsilon)\left(x_{1}-x\right)} .
$$

A straightforward calculation yields

$$
\lim _{x \rightarrow-\infty} \frac{-x}{1+F\left(x_{1}\right)+(L+\epsilon)\left(x_{1}-x\right)}=\frac{1}{L+\epsilon},
$$

therefore, choosing $\eta>0$, we can find $x_{2}<x_{1}$ such that for $x<x_{2}<0$,

$$
\frac{-x}{1+F\left(x_{1}\right)+(L+\epsilon)\left(x_{1}-x\right)}>\frac{1}{L+\epsilon}-\eta \text {. }
$$

Then, for $x<x_{2}<x_{1}<0$,

$$
\Gamma_{+}(x)=\Gamma_{+}\left(x_{2}\right)+\int_{x}^{x_{2}} \frac{-x}{1+F_{+}(x)}>\Gamma_{+}\left(x_{2}\right)+\left(x_{2}-x\right)\left[\frac{1}{L+\epsilon}-\eta\right] .
$$

At last

$$
\begin{aligned}
\lambda \Gamma_{+}(x)-F(x)> & \lambda \Gamma_{+}\left(x_{2}\right)+\lambda\left(x_{2}-x\right)\left[\frac{1}{L+\epsilon}-\eta\right]-F\left(x_{1}\right)-(L+\epsilon)\left(x_{1}-x\right) \\
= & x\left(L+\epsilon-\lambda\left[\frac{1}{L+\epsilon}-\eta\right]\right) \\
& +\left\{\lambda \Gamma_{+}\left(x_{2}\right)+\lambda\left(x_{2}\right)\left[\frac{1}{L+\epsilon}-\eta\right]-F\left(x_{1}\right)-x_{1}(L+\epsilon)\right\} .
\end{aligned}
$$

Then limit (2.22) is verified provided that

$$
L+\epsilon-\lambda\left[\frac{1}{L+\epsilon}-\eta\right]<0 .
$$

$\epsilon$ and $\eta$ being arbitrary, the existence of $0<\lambda<4$ such that the last inequality holds is ensured by $L<2$. With a very similar calculation (with $F_{+}(x)=F(x)-F(\alpha)$, where $\alpha$ is the non-null zero of $F$ ), hypothesis (2.16) yields to prove that

$$
\limsup _{x \rightarrow+\infty}\left[4 \Gamma_{+}(x)-F(x)\right]<+\infty .
$$

As the necessary condition for the intersection of the negative semi-trajectory with the vertical isocline is denied, there exists a point $Q=\left(x_{0}, y_{0}\right)$ such that $\gamma^{-}(Q)$ is not intersecting the curve $y=F(x)$ defined in (2.14). By a standard inspection of the direction of 


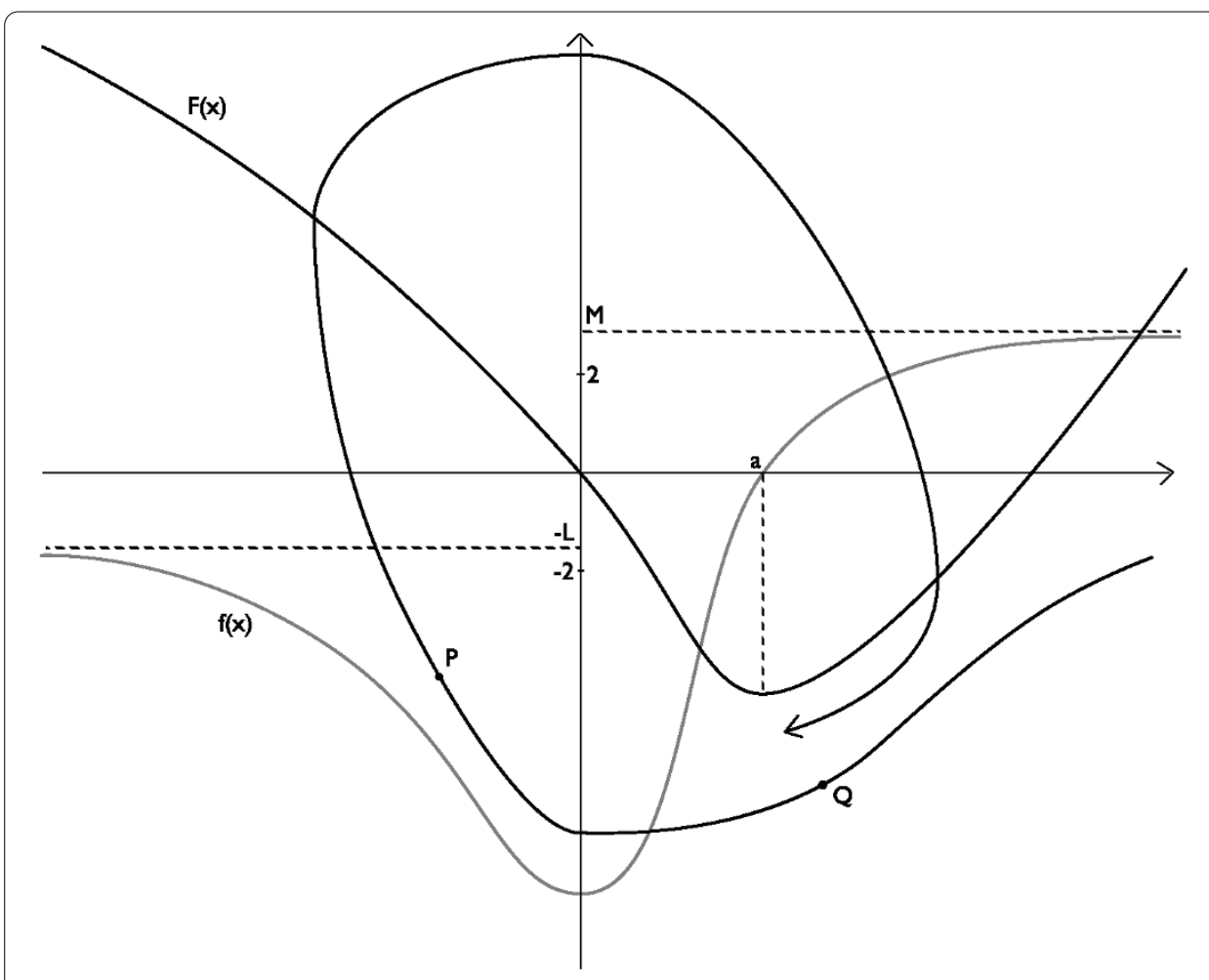

Figure 2 Trajectory $\gamma(Q)$ in Theorem 2.3.

the field, $\gamma^{+}(Q)$ crosses the negative $y$-semiaxis and reaches a point $P=\left(x_{1}, y_{1}\right)$ verifying (2.18). By the existence of $0<\lambda<4$ such that the sufficient condition (2.22) holds, the positive semi-trajectory $\gamma^{+}(P)$ intersects at first the $y$-axis because again there are no vertical asymptotes and then the curve $y=F(x)$ because the sufficient condition for intersection is fulfilled. By the uniqueness for the solutions to the Cauchy problem associated to system (1.3), trajectories cannot intersect and hence $\gamma^{+}(Q)$ is a winding trajectory (see Figure 2). Being $f(0)<0$, the origin is a source and the existence of a limit cycle is granted by the Poincaré-Bendixson theorem, while the uniqueness is given by monotonicity properties in virtue of the Massera theorem.

If we relax the monotonicity assumptions, we lose uniqueness, but existence still holds. Hence we have the following corollary.

Corollary 2.6 The Liénard system (1.3) has at least a stable limit cycle provided that $f$ is continuous and $f(x)(x-a)>0$ with $a>0$, and

1.

$$
\lim _{x \rightarrow-\infty} f(x)=-L>-2
$$

2.

$$
\lim _{x \rightarrow+\infty} f(x)=M>2 .
$$


Now we consider the case of non-existence of limit cycles. We consider again the results of [9] and, in particular, the case in which the semi-trajectory $\gamma^{-}(P)$, starting at a point $P=\left(x_{0}, y_{0}\right)$ verifying (2.19), intersects the vertical isocline in the positive $x$-half plane, but $\gamma^{+}(P)$ does not intersect the same curve in the negative $x$-half plane, and we get the following necessary condition.

Theorem 2.7 The Liénard system (1.3) has no limit cycles provided that $f$ is continuous and

1. $f(x)$ is monotone decreasing for $x<0$,

2. $f(x)$ is monotone increasing for $x>0$ and has a single zero at $a>0$,

3.

$$
\lim _{x \rightarrow-\infty} f(x)=-L<-2
$$

4.

$$
\lim _{x \rightarrow+\infty} f(x)=M<2
$$

Proof At first we need the following results.

Theorem 2.8 ([9, Theorem 2.6.]) If there is a constant $0<\lambda<4$ such that

$$
\lim _{x \rightarrow+\infty}\left[\lambda \Gamma_{+}(x)-F(x)\right]=+\infty
$$

then, for every $P=\left(x_{0}, y_{0}\right)$ verifying $(2.19), \gamma^{-}(P)$ intersects the curve $y=F(x)$ at $(x, F(x))$ with $x>x_{0}$.

Theorem 2.9 ( $\left[9\right.$, Theorem 2.2]) If, for every $P=\left(x_{0}, y_{0}\right)$ verifying $(2.18), \gamma^{+}(P)$ intersects the curve $y=F(x)$, then

$$
\limsup _{x \rightarrow-\infty}\left[4 \Gamma_{+}(x)-F(x)\right]=+\infty
$$

As in the previous theorem, the assumptions (2.28) and (2.29) become respectively

$$
\begin{aligned}
& \lim _{x \rightarrow+\infty}\left[\lambda \int_{0}^{x} \frac{\xi d \xi}{1+F(\xi)-F(\alpha)}-F(x)\right]=+\infty, \\
& \lim _{x \rightarrow-\infty}\left[4 \int_{0}^{x} \frac{\xi d \xi}{1+F(\xi)}-F(x)\right]=+\infty
\end{aligned}
$$

where $\alpha$ is the non-null zero of $F$.

With the same calculation, which we omit for the sake of simplicity, we get that if assumptions hold, we can provide a trajectory which is unwinding and goes to infinity without intersecting the curve $y=F(x)$ for $x$ negative (see Figure 3).

Now assume, by contradiction, that there exists a limit cycle. The monotonicity property of the Massera theorem guarantees that such a limit cycle is unique and stable. Therefore there are trajectories surrounding the limit cycle which are winding and going to such a 


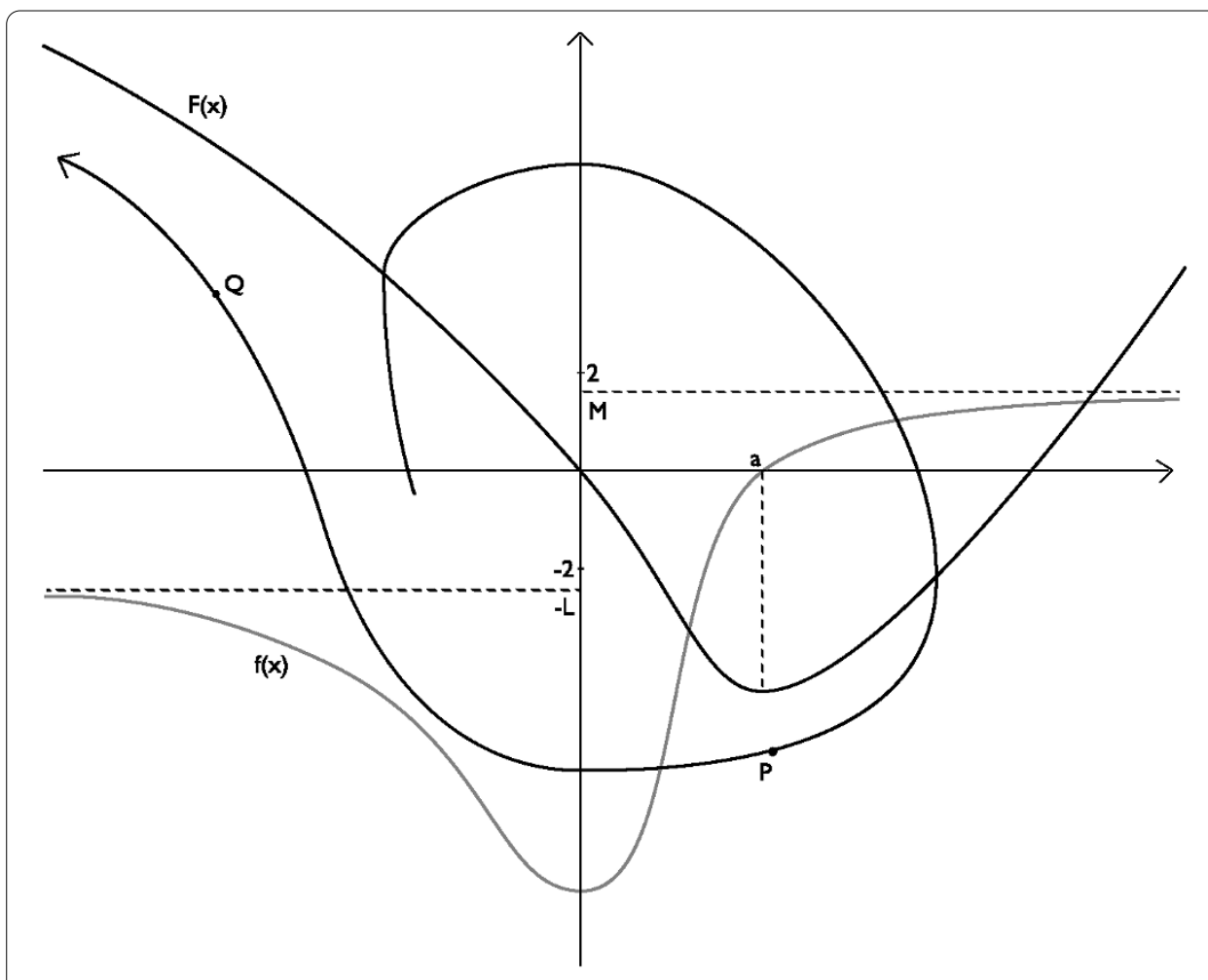

Figure 3 Trajectory $\gamma(Q)$ in Theorem 2.7.

cycle. But with the same calculation already applied, which we omit for the sake of simplicity, if assumptions (2.26) and (2.27) hold, we can provide a large trajectory which is unwinding and goes to infinity without intersecting the curve $y=F(x)$ for $x$ negative. We can apply again the Poincaré-Bendixson theorem and get the existence of a second limit cycle which is unstable.

But as already mentioned, the monotonicity properties of the Massera theorem give the uniqueness, and hence in this situation we can conclude that there is no limit cycle and all trajectories are unwinding.

We observe that there are cases which still are not investigated. For instance, if we consider $f(x)$ such that

$$
\lim _{x \rightarrow-\infty} f(x)=-L<-2 \lim _{x \rightarrow+\infty} f(x)=M>2,
$$

we can produce a trajectory which lies entirely below the curve $y=F(x)$ in the Liénard plane (or below the $x$-axis in the phase-plane) and therefore is not winding or unwinding. In order to describe in full the possible configurations, we consider the following example.

Example 2.10 Let $f$ be continuous, monotone decreasing for $x<0$ and monotone increasing for $x>0$ with a single zero at $a>0$.

Let $f(0)=-\alpha<0$, and

$$
\lim _{x \rightarrow-\infty} f(x)=-L=-2, \quad \lim _{x \rightarrow+\infty} f(x)=M=2 .
$$


Define $f_{\epsilon}(x)=f(x)+\epsilon$ and consider the system

$$
\left\{\begin{array}{l}
\dot{x}=y-F_{\epsilon}(x), \\
\dot{y}=-x,
\end{array} \quad \text { where } F_{\epsilon}(x)=\int_{0}^{x} f_{\epsilon}(t) d t .\right.
$$

If $\epsilon \in(-\infty, 0]$ or $[\alpha,+\infty)$, system (2.30) has no limit cycles, while if $\epsilon \in(0, \alpha)$, system (2.30) has exactly one limit cycle which is stable.

This is because if $-2<\epsilon<0$, we can apply Theorem 2.7, while if $\epsilon \leq-2$, then $f_{\epsilon}(x)$ is negative, and if $\epsilon>\alpha$, then $f_{\epsilon}(x)$ is positive (for $\epsilon=\alpha, f_{\epsilon}(x)$ is positive for $x \neq 0$ ). And as already mentioned, if $f_{\epsilon}$ does not change sign, there is no limit cycle. On the other hand, if $\epsilon \in(0, \alpha)$, then we can apply Theorem 2.3, and in this case there is a unique limit cycle which is stable.

We can describe the movement of such a cycle. At $\epsilon=\alpha, f_{\epsilon}(0)$ changes sign and we have a standard Hopf bifurcation, which gives a small amplitude limit cycle bifurcating from the origin. When $\epsilon$ decreases, such a limit cycle enlarges and eventually collapses to a separatrix at $\epsilon=0$. For a complete description of the bifurcation from a separatrix, we recall [10]. For the bifurcation of limit cycles we recall [11].

Competing interests

The authors declare that they have no competing interests.

Authors' contributions

Both authors contributed equally in writing this paper.

\section{Acknowledgements}

We thank the anonymous referees for their valuable suggestions and Dr. Francesco Della Santa for the figures.

Received: 14 December 2012 Accepted: 25 May 2013 Published: 11 June 2013

\section{References}

1. Carletti, T, Rosati, L, Villari, G: Qualitative analysis of the phase portrait for a class of planar vector fields via the comparison method. Nonlinear Anal. 67(1), 39-51 (2007)

2. Massera, JL: Sur un Théoreme de G. Sansone sur l'equation de Liénard. Boll. Unione Mat. Ital. 9(3), 367-369 (1954)

3. Sansone, G: Soluzioni periodiche dell'equazione di Liénard. Calcolo del periodo. Rend. Semin. Mat. (Torino) 10, 155-171 (1951)

4. Sabatini, M, Villari, G: On the uniqueness of limit cycles for Liénard equation: the legacy of G. Sansone. Matematiche LXV(II), 201-214 (2010)

5. Villari, G: An improvement of Massera's theorem for the existence and uniqueness of a periodic solution for the Liénard equation. Rend. Ist. Mat. Univ. Trieste 44, 187-195 (2012)

6. Villari, G: On the existence of periodic solutions for Liénard's equation. Nonlinear Anal. TMA 7, 71-78 (1983)

7. Levinson, N, Smith, O: A general equation for relaxation oscillations. Duke Math. J. 9, 382-403 (1942)

8. Villari, G: Periodic solutions of Lénard's equation. J. Math. Anal. Appl. 86, 379-386 (1982)

9. Villari, G, Zanolin, F: On a dynamical system in the Liénard plane. Necessary and sufficient conditions for the intersection with the vertical isocline and applications. Funkc. Ekvacioj 33, 19-38 (1990)

10. Villari, G, Villarini, M: Limit cycles and bifurcation from a separatrix for a polynomial Liénard system in the plane. Differ Equ. Dyn. Syst. 5, 423-437 (1997)

11. Perko, LM: Bifurcation of limit cycles: geometric theory. Proc. Am. Math. Soc. 114, 225-236 (1992)

doi:10.1186/1687-2770-2013-144

Cite this article as: Rosati and Villari: On Massera's theorem concerning the uniqueness of a periodic solution for the Liénard equation. When does such a periodic solution actually exist?. Boundary Value Problems 2013 2013:144. 